\title{
Endoscopic gastroplasty as therapy for postoperative gastric distortion and stenosis
}

Gastric stricture resulting in impairment of gastric emptying may occur after gastric surgery or laparoscopic surgery [13]. In some instances further surgery can be avoided by endoscopic balloon dilation of the stricture $[4,5]$. We present an unusual case of gastric distortion and stricture after resection of a gastrointestinal stromal tumor (GIST). Because balIoon dilation failed to effectively relieve the obstruction, endoscopic gastroplasty was performed with good success.

A 54-year-old man presented to our hospital with chronic abdominal distension and intermittent vomiting. He had undergone wedge resection of a GIST locat- ed in the greater curvature 5 months previously. After resection he began to experience abdominal pain and vomiting, and a gastric stricture was diagnosed. Endoscopic balloon dilation was performed, without improvement. He was then transferred to our hospital because of worsening symptoms. Upper endoscopy revealed gastric distortion and a stricture between the body and the antrum of the stomach ( $\triangleright$ Fig. 1 a, b). Abdominal computed tomography showed thickening of the distal stomach with narrowing of the lumen. After multidisciplinary team discussion we performed an endoscopic gastroplasty.
An endoscope with a transparent cap was inserted into the stomach and the stomach was washed with sterile saline. The area with the gastric scar (i. e., the greater curvature) was then marked with a hook knife. The hook knife and an insulated-tip knife were used to incise the mucosa longitudinally along the markers. Insulated-tip or hook knives were then used to complete full-thickness incision of the gastric wall on the greater curvature of the stomach ( $\mathbf{F i g . 1 C}$ ). Hemostasis was obtained with endoscopic electric coagulation. The incision was then closed transversely using endoclips and endoloops ( Fig.1d) as follows. First a nylon loop
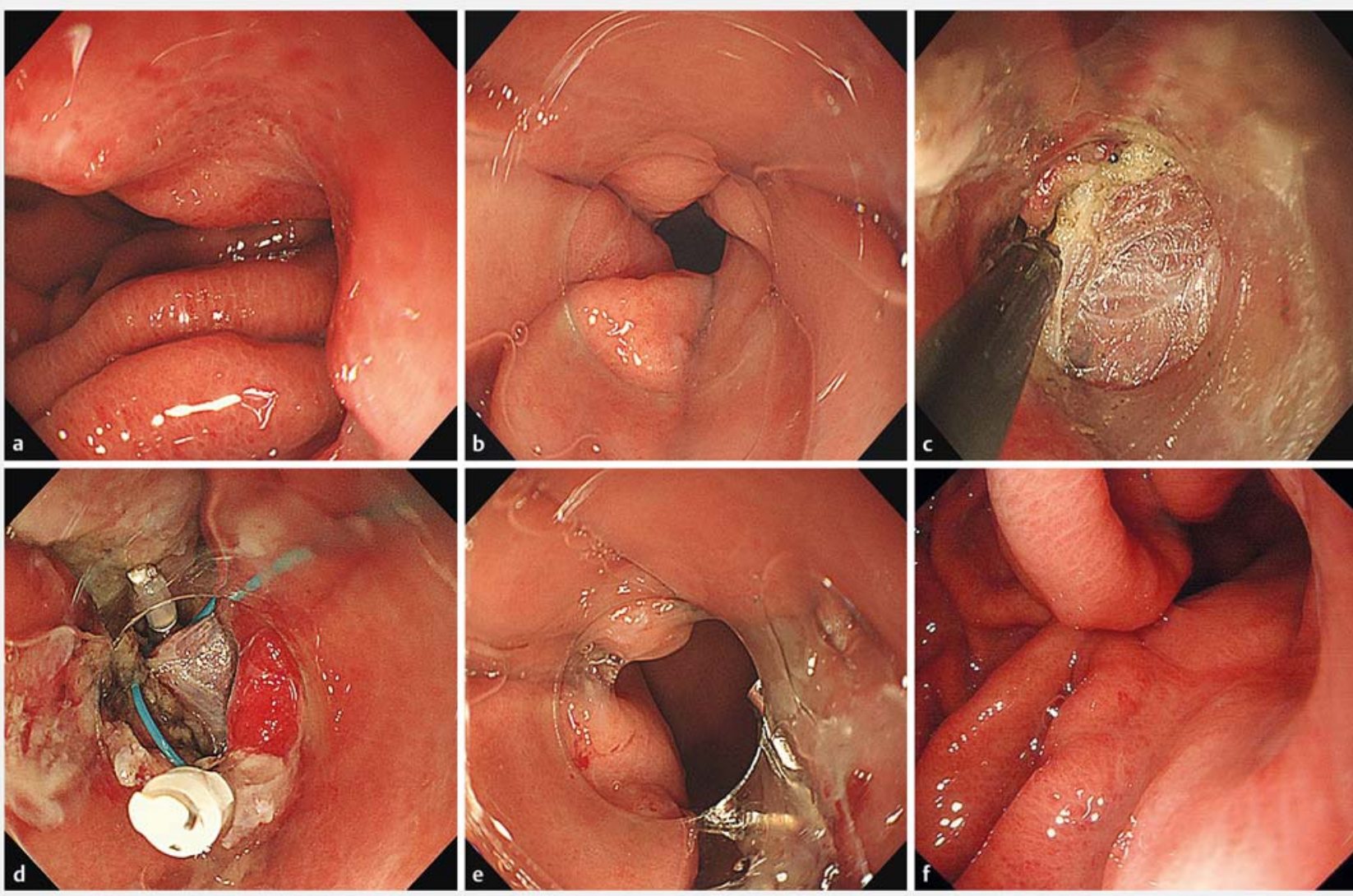

- Fig. 1 a, b Upper endoscopy revealed gastric distortion and stricture, 5 months after wedge resection of a gastrointestinal stromal tumor (GIST) in the greater curvature. c Full-thickness incision of the gastric wall on the greater curvature. $\mathbf{d}$ A nylon loop was fixed to the distal and proximal edges of the incision to close it transversely. e Endoscopy showed disappearance of the distortion and stricture after the procedure. f A widened lumen without distortion is seen 3 months postoperatively. 


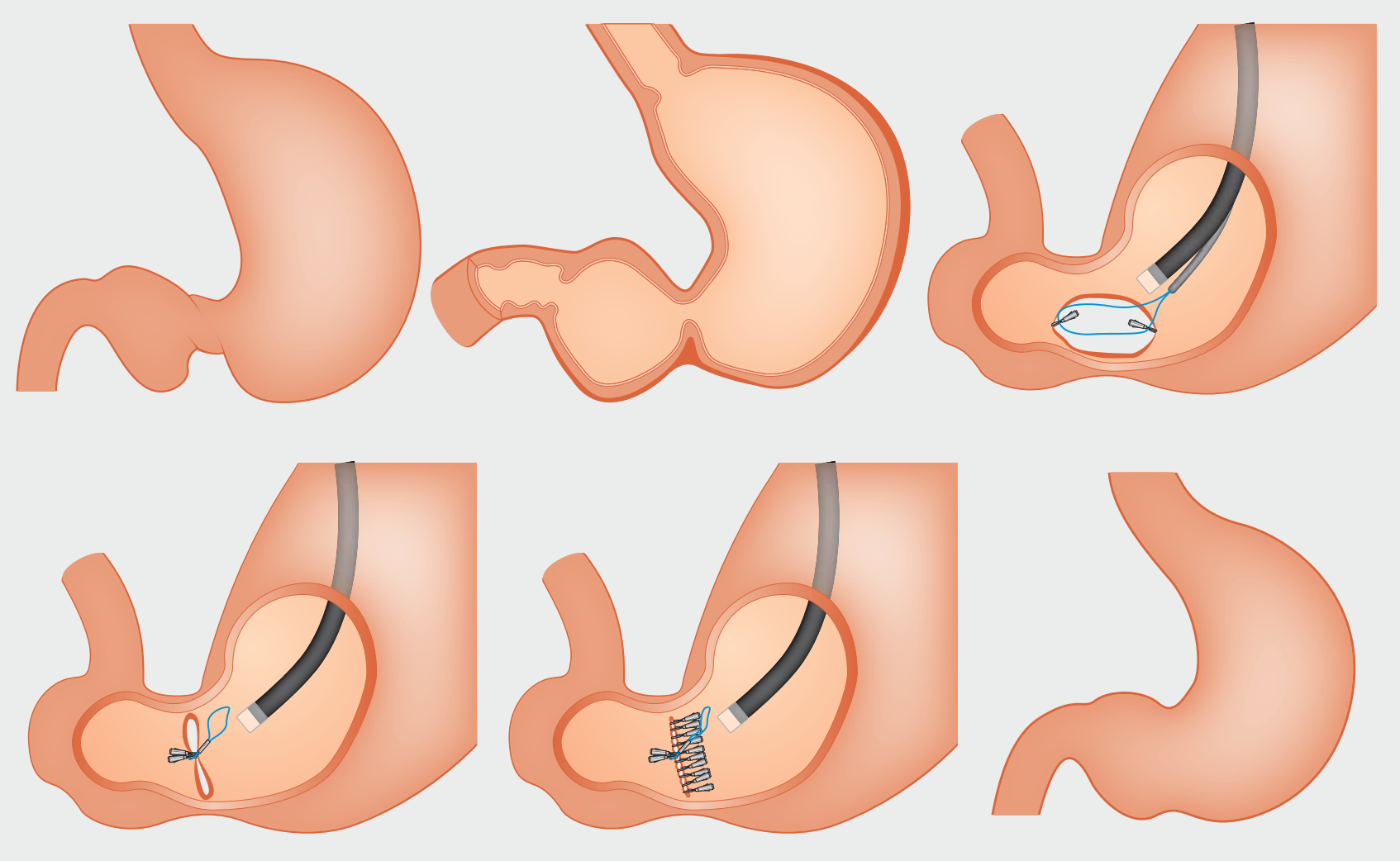

Fig. 2 Schematic of the endoscopic gastroplasty procedure and transverse closure method.

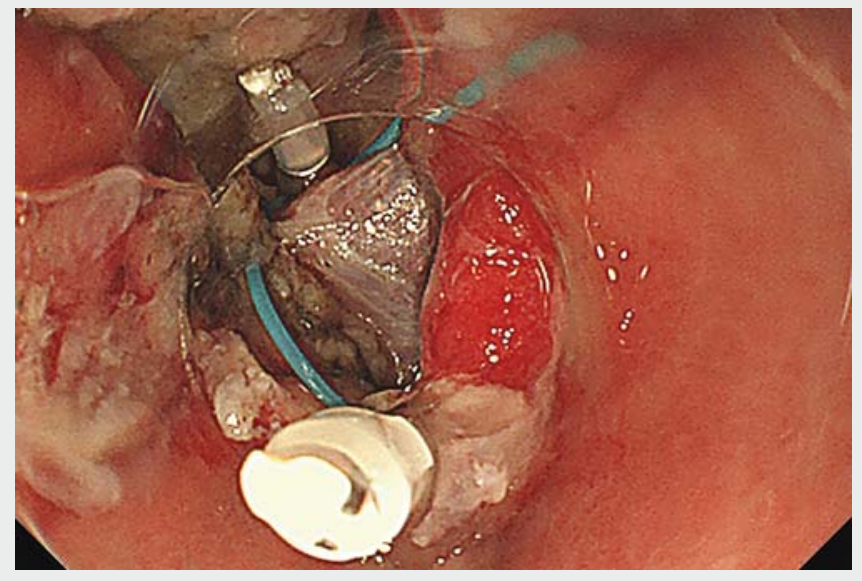

after the procedure. After the procedure, the endoscope was able to pass freely through the widened gastric lumen and the distortion had disappeared ( $\triangleright$ Fig.1e). No adverse events were noted during the follow-up period. It was found that the lumen had widened and gastric distortion had disappeared at endoscopy 3 months after the procedure ( Fig.1f).

We successfully performed endoscopic gastroplasty with results similar to those usually only obtained by surgery. This case suggests that that endoscopic fullthickness longitudinal incision with transverse gastric wall closure may be a minimally invasive, safe, feasible, and effective technique for management of patients with benign gastric stricture. Further validations are needed.

Video
stenosis.

was fixed on the transparent cap attached to the endoscope, passed to the incision site, and placed into the incision area. A clip was then used to fix one side to the distal edge of the full-thickness incision and a second clip was used to further anchor the nylon loop to the proximal edge of the incision. The nylon loop was then closed thus approximating the distal and proximal gastric walls to transform the oval-shaped longitudinal incision into a transverse gastric wall closure. Clips were then used to ensure complete closure of the incision ( $\triangleright$ Fig. 2, $\triangleright$ Video 1 ). No significant bleeding or any other complications occurred either during or
Endoscopy_UCTN_Code_TTT_1AO_2AN

\section{Acknowledgment}

We gratefully acknowledge the critical review of Professor David Y. Graham, MD Professor of Medicine, Molecular Virology and Microbiology, Baylor College of Medicine. 
None

The authors

Bing-Rong Liu, Dan Liu, Li-Xia Zhao, Saif Ullah, Yang-Yang Zhou, Ji-Yu Zhang, Kong Ling-Jian Department of Gastroenterology, First Affiliated Hospital of Zhengzhou University, Zhengzhou, Henan, China

\section{Corresponding author}

Bing-Rong Liu, MD, PhD

Department of Gastroenterology, First Affiliated Hospital of Zhengzhou University, 1 Eastern Jianshe Road, Zhengzhou, Henan 450052, China

bingrongliu@qq.com

Fax: +86-132-23017172
[1] Zundel N, Hernandez JD, Galvao Neto M et al. Strictures after laparoscopic sleeve gastrectomy. Surg Laparosc Endosc Percutan Tech 2010; 20: 154-158

[2] Uchida K, Ohtake K, Inoue M et al. Stapled gastroplasty for antral stricture after repair of neonatal gastric rupture: report of a case. Pediatr Surg Int 2005; 21: $331-332$

[3] Goldenberg A, Sandau R, Fakulujo A. EGD assisted stapled strictureplasty for gastric stricture after GIST tumor resection: a case report. Surgical Sci 2014; 5: $201-205$

[4] Holt PD, de Lange EE, Shaffer HA Jr. Strictures after gastric surgery: treatment with fluoroscopically guided balloon dilatation. AJR Am J Roentgenol 1995; 164: 895-899

[5] Vance PL, de Lange EE, Shaffer HA Jr et al. Gastric outlet obstruction following surgery for morbid obesity: efficacy of fluoroscopically guided balloon dilation. Radiology 2002; 222: 70 - 72

\section{Bibliography}

DOI https://doi.org/10.1055/a-0837-0583

Published online: 5.3.2019

Endoscopy 2019; 51: E105-E107

(c) Georg Thieme Verlag KG

Stuttgart · New York

ISSN 0013-726X

\section{ENDOSCOPY E-VIDEOS}

https:/|eref.thieme.de/e-videos

口回 Endoscopy E-Videos is a free access online section, reporting 田: on interesting cases and new techniques in gastroenterological endoscopy. All papers include a high quality video and all contributions are freely accessible online.

This section has its own submission website at https://mc.manuscriptcentral.com/e-videos 